\title{
Significados atribuídos ao envelhecimento: idoso, velho e idoso ativo
}

\author{
Olivia Galvão Lucena Ferreira - Faculdade de Ciências Médicas da Paraíba, João Pessoa, Brasil \\ Silvana Carneiro Maciel - Universidade Federal da Paraíba, João Pessoa, Brasil \\ Antonia Oliveira Silva - Universidade Federal da Paraíba, João Pessoa, Brasil \\ Roseane Christina da Nova Sá - Universidade Federal de Campina Grande, Campina Grande, Brasil \\ Maria Adelaide Silva P. Moreira - Universidade Estadual do Sudoeste da Babia, Jequié, Brasil
}

\begin{abstract}
Resumo
O presente estudo teve como objetivo apreender os significados atribuídos ao envelhecimento elaborados por idosos funcionalmente independentes. Utilizando o aporte teórico das representações sociais, tratou-se de priorizar as falas dos participantes, a fim de salientar as dimensões simbólicas acerca do envelhecimento. $O$ estudo foi realizado na Unidade de Saúde da Família (USF) localizada no bairro Alto do Mateus, na cidade de João Pessoa, PB. A amostra foi constituída por 100 idosos funcionalmente independentes, com idades entre 60 e 93 anos. Como instrumentos para a coleta de dados foram utilizados o MIF e o Teste de Associação Livre de Palavras, sendo os resultados submetidos à análise do software Tri-Deux-Mots. Com base nos resultados, observou-se que os estímulos idoso e velho foram associados a aspectos com conotações mais negativas. No entanto, para o estímulo idoso ativo, embora existissem as conotações negativas, os significados positivos foram mais enfocados pelos idosos investigados.

Palavras-chave: Envelhecimento, Idoso, Idoso ativo.
\end{abstract}

\section{Meanings assigned to aging: elderly, old and active elderly}

\begin{abstract}
This present study intended to understand the representations produced by a group of functionally independent aged people about the ageing process. Starting from the theoretical principles of social representations, priority was given to the participants' speech in order to stress the symbolical dimensions about ageing. The study was carried out at a family health unit in "Alto do Mateus", a suburban area of "João Pessoa", "Paraíba". The sample included 100 functionally independent aged persons, from 60 to 93 years old. Data were collected through the "MIF" and the test of Free Association of Words; and the results were analyzed by means of the "Tri-Deux-Mots" software. The results showed that the aged and the old stimuli were connected with aspects of more negative connotation. However, for the "active elderly" stimulus, though the negative connotation still subsisted, the positive significations showed more evidence among the aged persons under investigation.

Keywords: Ageing process, Elderly, Active elderly.
\end{abstract}

A literatura científica apresenta distintos conceitos para o envelhecimento. Tais conceitos têm considerado diferentes aspectos do desenvolvimento humano, passando pelos campos biológico, social, psicológico e cultural. Contudo, ainda não é possível encontrar uma definição de envelhecimento que envolva os complicados caminhos que levam o indivíduo a envelhecer e como este processo é vivenciado e representado pelos próprios idosos e pela sociedade em geral (Carvalho Filho \& Papaléo Netto, 2006; Uchôa, 2003).

O envelhecimento é um fenômeno do processo da vida, assim como a infância, a adolescência e a maturidade, e é marcado por mudanças biopsicossociais específicas, associadas à passagem do tempo. No entanto, este fenômeno varia de indivíduo para indivíduo, podendo ser determinado geneticamente ou ser influenciado pelo estilo de vida, pelas características do meio ambiente e pela situação

\footnotetext{
${ }^{1}$ Endereço para correspondência:

Rua Vereador Gumercindo Barbosa Dunda, 378, apto 1.401 58036-850 - João Pessoa/PB
}

nutricional de cada um (Ávila, Guerra \& Meneses, 2007).

Diversidades também são encontradas nas representações e significados atribuídos ao envelhecimento e ao idoso. Estudos como os de Lopes e Park (2007) destacam que, atualmente, percebe-se, por um lado, imagens de velhos que procuram manter o controle sobre seus corpos e relativa juventude, ativos e dispostos a realizar sonhos e satisfazer seus desejos; e, por outro, imagens de velhos pobres, doentes, solitários, assexuados e abandonados à sua sorte. Desse modo, coexistem diferentes imagens de velhos na sociedade contemporânea.

Nesse contexto, a Teoria das Representações Sociais, cuja contribuição tem sido significativa para a compreensão de diversos fenômenos, apresenta-se como um referencial importante para o estudo dos significados atribuídos ao envelhecimento e ao idoso. No que concerne aos idosos, a exploração das suas representações poderá permitir o contato com imagens e conteúdos que expressam, de certa forma, as suas necessidades psicossociais. Este aspecto torna-se determinante para a construção de condutas e o planejamento de ações que promovam um 
envelhecimento saudável e bem sucedido. (Ávila e colaboradores, 2007)

$\mathrm{Na}$ sociedade atual, diversos estudos sobre representações sociais revelam que a idéia de idoso está geralmente associada a aspectos negativos, como figura decadente, necessitada e dependente. Enquanto fenômeno psicossocial, essas concepções contribuíram para os processos de formação de condutas, orientação das comunicações sociais e estruturação da identidade do idoso, assim como para as práticas sociais a ele dirigidas. Portanto, o envelhecimento é marcado por diversas experiências, que são norteadas por valores, metas, crenças e formas próprias que o idoso utiliza para interpretar o mundo (Almeida \& Cunha, 2003).

Estudar o envelhecimento baseado em uma perspectiva psicossocial, ressaltando aspectos sociocognitivos, culturais e biológicos, entre outros, configura-se como uma importante vertente atual, na medida em que levam em conta os determinantes psicossociais que influenciam os posicionamentos das pessoas ante o idoso. Nessa perspectiva, o aporte teórico das representações sociais significa uma abordagem interessante e oportuna para se compreender e propor estratégias de atendimento ao idoso contextualizado socialmente. Certamente, esses aspectos contribuirão para a implementação de ações ou práticas profissionais mais assertivas no atendimento oferecido a essa população.

A Teoria das Representações Sociais (TRS) surgiu com os estudos de Moscovici na década de 60 (1961/1978), enfatizando a indissociação entre o individual e o social, construindo um novo campo de ação que estaria na intersecção desses dois: a psicossociologia, a qual significa uma forma de conhecimento prático, que funciona como mediadora entre sujeito e objeto, no contexto sociointeracional. Nesse sentido, essa teoria torna-se útil para a análise de diferentes objetos e fenômenos de estudo, particularmente no campo da saúde.

As representações sociais compreendem um sistema de interpretação da realidade constituído por processos sociocognitivos, com implicações nas relações dos indivíduos com o mundo, nas suas condutas e nos seus comportamentos no meio social (Herzlich, 2005). Em relação ao funcionamento das representações sociais, Moscovici evoca dois processos fundamentais: a ancoragem e a objetivação. Moscovici (2007) afirma que objetivar significa transformar uma noção abstrata em algo concreto, tornando-a palpável e objetiva, dando uma forma específica ao conhecimento que se tem a seu respeito com a finalidade de reproduzir algo não-familiar em algo que pode ser compreendido e controlado. Já a ancoragem é definida como o processo de classificar informações sobre um objeto social em relação a estruturas de conhecimento anteriormente existentes, ligando as representações sociais a uma memória coletiva, a contextualização histórica.

Moscovici (2009) destaca que, mais concretamente, tudo se ordena na representação a partir de um nó figurativo, que condensa todas as imagens, todas as noções ou os julgamentos que um grupo ou uma sociedade gera ao longo do tempo. As representações sociais são construídas com base nas informações que circulam pela sociedade, nas relações sociais e no movimento do grupo no qual nascem, transformam-se e podem se extinguir, sendo importante conhecer o contexto em que são produzidas para que sejam compreendidas (Jodelet, 2005).

Assim, avaliar os idosos no contexto das Unidades de Saúde da Família possibilita dimensionar um diagnóstico psicossocial importante para o seu atendimento, na medida em que é feito em um contexto singular e conhecendo as possíveis necessidades de atenção à saúde que garantam os seus direitos como cidadãos e a sua inclusão na sociedade, além de uma melhor qualidade de vida. É nessa direção que se insere o presente estudo, que tem o objetivo de apreender as representações e os significados associados ao envelhecimento, na concepção de idosos considerados como funcionalmente independentes.

\section{Método}

\section{Lócus}

O estudo foi realizado na Unidade de Saúde da Família (USF) Nova Conquista, que é uma unidade integrada, formada por quatro equipes de Saúde da Família, localizadas no bairro Alto do Mateus, na cidade de João Pessoa-PB.

\section{Participantes}

A amostra foi definida a partir da seleção de 100 idosos funcionalmente independentes, em condições físicas e mentais para responder os instrumentos. As condições dos idosos foram confirmadas pela Medida de Independência Funcional (MIF), que foi o instrumento de avaliação funcional utilizado na determinação do ponto de corte para a constituição da amostra.

A idade dos idosos variou entre 60 e 93 anos $(M=68 ; D P=7,53)$. A maioria era do sexo feminino $(73 \%)$, residente em casa própria $(88 \%)$, convivendo com o cônjuge e parentes próximos $(75 \%)$. Quanto à escolaridade, $40 \%$ indicaram não ser alfabetizados, $48 \%$ disseram ter o ensino fundamental incompleto, 
$8 \%$ o ensino médio e $4 \%$ o ensino superior, sendo $73 \%$ católicos, 24\% evangélicos e 3\% testemunhas de Jeová.

Ética

A participação dos idosos foi voluntária e atendeu à Resolução 196/1996, do Ministério da Saúde/Conselho Nacional de Saúde/Comissão Nacional de Ética em Pesquisa, que estabelece as Diretrizes e Normas que regem as pesquisas envolvendo seres humanos; tendo sido aprovada pelo Conselho de Bioética da UFPB $\backslash$ CCS (protocolo $\mathrm{n}^{\circ}$ $0188 \backslash 08)$. Por ocasião das entrevistas, os participantes preencheram e assinaram um termo de consentimento livre e esclarecido.

\section{Instrumentos}

Para a avaliação funcional dos idosos, foi adotado o instrumento preconizado pelo Ministério da Saúde, a Medida de Independência Funcional (MIF). Este instrumento tem como objetivo avaliar a independência funcional, medindo o grau de solicitação de cuidados de terceiros para a realização de tarefas motoras e cognitivas, tais como: autocuidado, controle de esfíncteres, transferência e locomoção, comunicação e cognição social. Foi avaliado o desempenho do idoso em atividades como: alimentação; higiene pessoal/matinal; banho; vestir-se acima da cintura; vestir-se abaixo da cintura; uso do vaso sanitário; controle de urina; controle das fezes; transferência do leito, cadeira e cadeira de rodas; vaso sanitário; chuveiro/banheira; locomoção/marcha; escadas; compreensão; expressão; interação social; resolução de problemas; e memória (Brasil, 2006).

Cada item foi classificado em uma escala de graus de dependência de 7 níveis, sendo o valor 1 correspondente à dependência total $\mathrm{e} O$ valor 7 correspondente à normalidade na realização de tarefas de forma independente. Os dados coletados a partir da Escala MIF e que serviram como ponto de corte para a seleção da amostra, foram analisados pelo software SPSS 15.

\section{Procedimentos}

Após a utilização do MIF, foi aplicado o Teste de Associação Livre de Palavras, que empregou os estímulos indutores idoso, velho e idoso ativo. Os resultados apreendidos a partir do Teste de Associação Livre de Palavras foram organizados em um banco de dados, tendo por base a construção de um dicionário de vocábulos concernente a cada estímulo indutor. Em seguida, procedeu-se a uma análise de conteúdo semântico, para possibilitar a codificação e a introdução dos dados no software Tri-Deux-Mots. O TriDeux-Mots é um programa informático que realiza uma análise fatorial de correspondência (AFC), sendo indicado para a análise de questões abertas, questões fechadas e dados relativos à associação de palavras.

O Tri-Deux-Mots representa graficamente as variações semânticas na organização do campo espacial, revelando aproximações e oposições das modalidades na construção dos fatores analisados pela Análise Fatorial de Correspodência (AFC). Por meio da AFC, torna-se possível verificar os vínculos existentes entre os diferentes conteúdos representacionais, correspondentes às respostas evocadas pelos sujeitos, denominadas de variáveis de opinião (respostas evocadas pelas três palavras-estímulo) e as variáveis fixas ou sociodemográficas, relativas às características de inserção social dos indivíduos (sexo, idade, escolaridade e procedência).

A análise fatorial produz cargas fatorias, as quais podem ser consideradas como pesos de regressão das variáveis mensuradas para predizer o construto subjacente. Quanto maior o valor da carga fatorial, melhor a variável representa o fator. Outro critério para as soluções fatoriais é estabelecer que os fatores precisam explicar uma percentagem significativa da variância total das variáveis medidas, devendo o conjunto de fatores explicar pelo menos $50 \%$ da variância comum.

A análise do plano fatorial é feita a partir do jogo de operações reveladas pelas respostas aos estímulos indutores, em função dos resultados da AFC. Essa análise permite mostrar uma estrutura mais central em relação a outros elementos do campo representacional e, portanto, a expressão da organização cognitiva a respeito de um objeto representado. $\mathrm{Na}$ literatura estatística sobre AFC, convencionou-se que o eixo 1 ou fator 1 deve ocupar a posição horizontal no gráfico, enquanto o eixo 2 ou fator 2 se mantém na vertical (Nóbrega, Fonte \& Paula, 2005).

\section{Resultados e discussão}

Após a construção do dicionário e a agregação dos conteúdos, os dados foram lançados no software TriDeux-Mots, para possibilitar a redução dos dados verbais volumosos num tempo reduzido. Ou seja, a análise fatorial reduziu a complexidade de uma grande quantidade de variáveis a uma estrutura relativamente simples, consistindo em um número menor de fatores, que são combinações lineares das variáveis observadas. Esse tipo de análise permitiu distinguir correlações (positivas e negativas) entre os diferentes grupos de pertença, do mesmo modo que foi possível apreender graficamente a atração entre as variáveis fixas e as 
variáveis de opiniões ou modalidades, que correspondem às palavras evocadas pelos sujeitos.

Os dados representados graficamente na Figura 1 referem-se aos estímulos idoso, velho e idoso ativo. Obteve-se um somatório igual a 1.394 palavras evocadas pelos participantes, das quais 269 são diferentes, representando as reações dos atores sociais em relação aos estímulos indutores. A análise dos dados coletados realizou-se mediante a leitura gráfica das representações, que estão distribuídas de maneira oposta sobre os dois fatores/eixos (F1 e $\mathrm{F} 2)$.

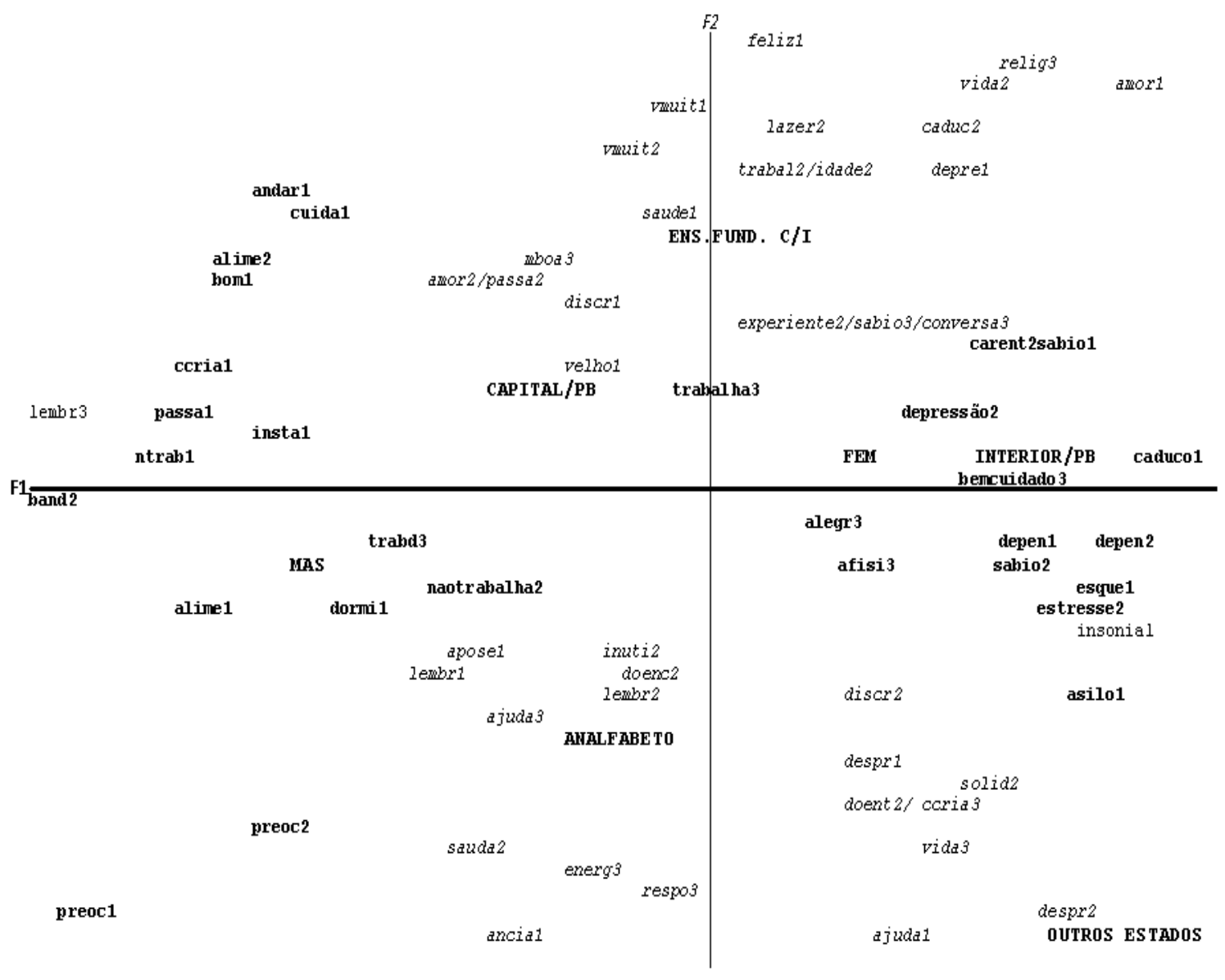

Figura 1- Plano Fatoria de Correspondência das Representações æcerca do idoso, velho e ido so ativo, Gaboradas palos idosos

O espaço fatorial, constituído pelos fatores F1 e F2 e delimitado pelas respostas aos três estímulos indutores (idoso, velho e idoso ativo) revela a existência de agrupamentos representacionais que evidenciam as semelhanças e diferenças nos conteúdos e na estrutura das representações dos atores sociais. Desse modo, no primeiro fator (F1), representado pelas palavras em negrito, na linha horizontal, encontram-se as representações de valor estatístico mais significativo, explicando $33,9 \%$ da variância total das respostas. Enquanto isso, o segundo fator (F2), na linha vertical representado pelas palavras em itálico, representa $25,5 \%$ da variância total das respostas. Pode-se verificar que a soma das variâncias perfaz um total de 59,4\%, permitindo concluir que os dados são estatisticamente significativos, ou seja, satisfatórios para a interpretação dos resultados.

No campo semântico do eixo $1-\mathrm{F} 1$, no centro e nas margens direita e esquerda, situaram-se as modalidades mais consensuais elaboradas pelos idosos. À direita, observa-se que, para os participantes do sexo feminino e oriundos do interior da Paraíba, o idoso (estímulo 1) foi considerado como "sábio", "caduco", "dependente" e "esquecido", sofrendo de "insônia" e lembrando o "asilo". O velho (estímulo 2) foi representado como um indivíduo "sábio", sem "estresse" e "carente", que sofre de "depressão" por ser "dependente". O idoso ativo (estímulo 3) foi representado como uma pessoa "alegre", que tem "trabalho", pratica uma "atividade física", é "bem cuidado" e tem "lembrança". 
$\grave{A}$ esquerda, ainda no eixo 1, encontram-se as representações dos participantes do sexo masculino e de procedência da capital da Paraíba. Eles associaram o idoso a alguém que "não trabalha", é "aposentado", tem "instabilidade", precisa de "cuidado" e de "alimentação certa". Além disso, o idoso foi associado a "criança" e "passado", sendo considerado como uma pessoa que "dorme muito", tem "lembranças" e "preocupação" e é um "andarilho". O velho foi considerado como "abandonado", que "não trabalha" e precisa de "alimentação", acarretando "preocupação". Enquanto isso, o idoso ativo foi associado ao "trabalho doméstico".

No campo semântico do eixo 2 - F2, na periferia superior, observa-se que os participantes com ensino fundamental e de procedência da capital/PB associaram o estímulo idoso a uma pessoa "feliz", que inspira "amor", tem "depressão", sofre "discriminação" e precisa cuidar da "saúde", pois é "velho" e "viveu muito". O velho foi associado a "vida", "lazer" e "trabalho", sendo considerado como "caduco", com "idade" e com um "passado", pois "viveu muito", é uma pessoa "experiente" e inspira "amor". O estímulo idoso ativo foi associado a "religião", "sábio", "conversa" e "memória boa".

No eixo 2, na margem inferior, verifica-se que os idosos analfabetos e de procedência de outros estados representaram o idoso como um "ancião", que necessita de "ajuda" e sofre "desprezo". O velho foi representado como "doente" e "inútil", centrado na "lembrança" e na "saudade repleta", que vivencia "doença" e sofre por "discriminação", "solidão" e "desprezo". O estímulo idoso ativo foi comparado a uma "criança", embora seja uma pessoa "responsável", que tem "energia", "ajuda" o próximo e é associado à própria "vida".

A partir da distribuição das palavras evocadas no plano fatorial de correspondência, pode-se observar que os estímulos idoso e velho foram associados a aspectos com conotações tanto positivas quanto negativas. No entanto, para o estímulo idoso ativo, embora possam ser notadas algumas conotações negativas, sobressaíram os significados mais positivos, no olhar dos idosos investigados.

$\mathrm{Na}$ Tabela 1 encontram-se as frequências das palavras mais evocadas, relativas aos estímulos indutores (idoso, velho e idoso ativo). As palavras associadas aos estímulos idoso e velho apresentam semelhanças e se referem, em geral, a aspectos negativos. Entretanto, em um estudo realizado sobre idoso e velhice, Ávila e colaboradores (2007) encontraram distinções entre essas categorias. $\mathrm{Na}$ primeira delas, os participantes idosos se reconheceram como velhos e relataram que a velhice não tem um momento certo para iniciar. Na categoria "velhice", os participantes se representaram como envelhecidos, mas não se consideraram como velhos.

Tabela 1 - Frequências relativas aos estímulos indutores idoso, velho, idoso ativo

\begin{tabular}{lll}
\hline $\begin{array}{l}\text { Estímulo } 1 \\
\text { "idoso" }\end{array}$ & $\begin{array}{l}\text { Estímulo } 2 \\
\text { "velho" }\end{array}$ & $\begin{array}{l}\text { Estímulo 3 } \\
\text { "idoso ativo" }\end{array}$ \\
\hline Abandonado $(\mathrm{F}=30)$ & Chato $(\mathrm{F}=60)$ & Atividade física $(\mathrm{F}=102)$ \\
Aposentado $(\mathrm{F}=30)$ & Depressão $(\mathrm{F}=39)$ & Alegre $(\mathrm{F}=105)$ \\
Cuidado $(\mathrm{F}=30)$ & Desrespeito $(\mathrm{F}=42)$ & Bom $(\mathrm{F}=39)$ \\
Depressão $(\mathrm{F}=36)$ & Discriminação $(\mathrm{F}=33)$ & Conversa $(\mathrm{F}=54)$ \\
Desprezo $(\mathrm{F}=51)$ & Doença $(\mathrm{F}=63)$ & Disposição $(\mathrm{F}=66)$ \\
Discriminado $(\mathrm{F}=30)$ & Doente $(\mathrm{F}=39)$ & Independência $(\mathrm{F}=126)$ \\
Doença $(\mathrm{F}=66)$ & Experiência $(\mathrm{F}=33)$ & Lazer $(\mathrm{F}=105)$ \\
Doente $(\mathrm{F}=39)$ & Limitação $(\mathrm{F}=84)$ & Memória boa $(\mathrm{F}=36)$ \\
Esquecimento $(\mathrm{F}=36)$ & Morte $(\mathrm{F}=42)$ & Trabalha $(\mathrm{F}=153)$ \\
Experiência $(\mathrm{F}=39)$ & Não trabalha $(\mathrm{F}=51)$ & Saúde $(\mathrm{F}=90)$ \\
Inútil $(\mathrm{F}=54)$ & Respeito $(\mathrm{F}=30)$ & \\
Limitação $(\mathrm{F}=90)$ & Triste $(\mathrm{F}=42)$ & \\
Respeito $(\mathrm{F}=33)$ & & \\
Velho $(\mathrm{F}=84)$ & & \\
\hline
\end{tabular}


Como pode ser observado na Tabela 1, a palavra "velho" foi associada ao estímulo idoso, o que pode justificar as semelhanças nas respostas. É interessante destacar a ocorrência, com maior frequência para este estímulo, da palavra "limitação". Ela aparece relacionada a alguma limitação funcional, como por exemplo: dificuldades para se alimentar, caminhar, aprender e jogar futebol; déficits de equilíbrio, visão e audição; fraqueza; restrições quanto a fumar, comer de tudo, viajar sozinho, pegar peso e fazer sexo; e medo de quedas.

Quanto ao estímulo velho, pode-se observar que a palavra "limitação" também apresentou a maior frequência, relacionando-se aos mesmos aspectos anteriormente citados. Entretanto, deve-se destacar o termo "morte", que surgiu como resposta a este estímulo com uma frequência igual a 42 .

Para o estímulo idoso ativo, observa-se uma associação significativa com os aspectos positivos do envelhecer, destacando-se os termos "trabalha", "independência", "lazer", "alegre" e "atividade física". Esses termos representam o idoso ativo como um indivíduo independente funcionalmente, que possui autonomia e bem-estar. Nesse sentido, Joia, Ruiz e Donalisio (2007) afirmam que "envelhecimento ativo", "envelhecimento bem sucedido" e "qualidade de vida na velhice" têm sido conceituados de maneira semelhantes, como satisfação pela vida.

Alguns estudos também apontam a mudança na representação da velhice e a formação de uma nova identidade para o idoso, ancorado em novos conceitos. Tais estudos fazem referência a uma velhice ativa, com possibilidades de realização, reforçando a importância da autonomia e da independência, o que se opõe ao tradicional discurso do envelhecimento passivo (Rosa, Benício, Latorre \& Ramos, 2003).

Ávila e colaboradores (2007) relatam que as imagens de tristeza, dores sem fim, isolamento e falta de perspectivas diante do envelhecimento vêm perdendo, aos poucos, os seus lugares no imaginário coletivo, sendo transformadas as representações acerca do envelhecimento e seus significados: idoso, velho e idoso ativo. O idoso está aprendendo a importância e a necessidade de adotar hábitos saudáveis, como forma de preservar e melhorar sua vida, saúde e bem-estar.

\section{Considerações finais}

De maneira geral, o envelhecimento pode ser definido pelos efeitos que a idade causa no organismo de uma pessoa. Nesse sentido, diz respeito a todas as consequências que acontecem desde o seu nascimento, caracterizando um processo que é construído ao longo de toda a sua vida (Ávila e colaboradores, 2007).
No presente trabalho, foi possível observar que a representação do envelhecimento, sem estar associado à palavra "ativo", foi baseada em aspectos negativos, ancorados em termos como velho, limitação, doença, inútil. Apesar de não ser sinônimo de doença, o envelhecimento acarreta alterações anatomopatológicas, que geralmente se apresentam através de doenças crônicas. Assim, o envelhecimento tem sido associado a uma prevalência de doenças crônicas, causadoras de dependência, fragilidades, incapacidades e morte (Garcia e colaboradores,2006; Pacheco \& Santos,2004).

Segundo Uchôa (2003), essa visão negativa e deficitária do envelhecimento, que é característica do ocidente, pode ser explicada como consequência de uma sociedade centrada na produção, no rendimento, na juventude e no dinamismo. Em sociedades nãoocidentais, o envelhecimento é geralmente apresentado por imagens bem mais positivas, mostrando que uma representação centrada em aspectos negativos não é universal.

Logo, o processo de envelhecimento precisa deixar de ser visto como uma decorrência de fenômenos puramente naturais e biológicos. Ele deve ser visualizado, também, como um fenômeno profundamente influenciado pela cultura, onde os indivíduos reagem a partir de suas referências pessoais e culturais.

$O$ fato de o envelhecimento continuar sendo representado sob a forma de perdas, faz com que muitas capacidades que as pessoas idosas possuem permaneçam desconhecidas e muitos ganhos não sejam valorizados. Nesse sentido, Caldas (2003) ressalta a importância da implementação de novas políticas públicas direcionadas aos idosos, que não apenas interfiram em aspectos relacionados à saúde, mas permitam o combate ao preconceito de que geralmente são vítimas, incentivem a sua valorização e a sua inserção na sociedade. Deve-se buscar a quebra do paradigma do envelhecimento objetivado na figura de velho e idoso, vinculado a doença, inutilidade e limitação; o novo paradigma deve estar ancorado na representação de idoso ativo, o qual vem associado a representações positivas de saúde, independência, alegria. Intrinsecamente relacionada com esta questão, torna-se evidente a necessidade tanto de qualificação dos profissionais voltados especificamente para o atendimento ao idoso, quanto de fiscalização por parte dos órgãos representativos dos profissionais de todas as áreas. Para Caldas (2003), é reconhecida a importância de políticas públicas direcionadas aos idosos que não apenas interfiram em aspectos relacionados à saúde, mas que permitam o combate ao preconceito, a valorização e a inserção na sociedade e 
devem ser comprometidas, responsáveis e com empenho em sua implementação.

Faz-se necessária, também, uma educação para a velhice nos diversos espaços, que pode ser alcançada com a construção social de uma imagem mais positiva do envelhecimento. Isso poderá ser iniciado no ambiente familiar, uma vez que o suporte da família e o convívio com os entes queridos são entendidos como fatores primordiais para o desenvolvimento de um envelhecimento saudável, que pode ser estimulado pela participação do idoso na vida cotidiana. Nas escolas, podem ser proporcionados encontros intergeracionais, por meio de rodas de conversas e espaços de trocas com idosos da comunidade. Esses encontros podem também favorecer a inclusão de temas sobre $\mathrm{O}$ processo de envelhecimento nos currículos das escolas. No trabalho, devem ser valorizadas as capacidades, as habilidades profissionais e, principalmente, as experiências adquiridas durante o decorrer da vida. Ao lado disso, deve existir uma preparação adequada para a aposentadoria.

Envelhecer é um privilégio para aqueles que alcançam essa etapa da vida. Por isso, a valorização das experiências vivenciadas no decorrer da existência dos idosos transforma-os em autoridades históricas para a transmissão de erros e acertos, que podem contribuir para a formação de uma consciência crítica sobre o presente.

\section{Referências}

Almeida, A. M. O. \& Cunha, G. G. (2003). Representações sociais do desenvolvimento humano. Psicologia: Reflexão e Crítica, 16(1),147-155.

Ávila, A. H., Guerra M. \& Meneses M. P. R. (2007). Se o velho é o outro, quem sou eu? A construção da auto-imagem na velhice. Pensamento Psicológico, 3(8), 7-18.

Brasil (2006). Ministério da Saúde. Secretaria de Atenção à Saúde. Departamento de Atenção Básica. Envelhecimento e saúde da pessoa idosa. Caderno de Atenção Básica. Brasília, DF, 19, 48-167.

Caldas, C. P. (2003). Envelhecimento com dependência: responsabilidades e demandas da família. Cadernos de Saúde Pública, 19(3), 773-781.

Carvalho Filho, E. T. \& Papaléo Netto, M. (2006). Geriatria: fundamentos, clínica e terapêutica. ( $2^{\mathrm{a}}$ ed.). São Paulo: Atheneu.
Garcia, M. A. A., Yagi, G. H., Souza, C. S., Odoni, A. P. C., Frigério, R. M. \& Merlin, S. S. (2006). Atenção à saúde em grupos sob a perspectiva dos idosos. Revista Latino-Americana de Enfermagem; 14(2),175-182.

Herzlich, C. (2005). Problemática da representação social e sua utilidade no campo da doença. PHYSIS: Revista Saúde Coletiva, 15(2), 57-70.

Jodelet, D. (2005). Loucuras e representações sociais. Petrópolis: Vozes.

Joia, L. C., Ruiz, T. \& Donalisio, M. R. (2007). Condições associadas ao grau de satisfação com a vida entre a população de idosos. Revista de Saúde Pública, 41(1), 131-38.

Lopes, E. S. L. \& Park, M. B. (2007). Representação social de crianças acerca do velho e do envelhecimento. Estudos de Psicologia, 12(2), 141 148.

Moscovici, S. (1961/1978). A representação social da psicanálise. Rio de Janeiro: Zahar.

Moscovici, S. (2007). Representações sociais. investigações em psicologia social. Petrópolis,RJ: Vozes.

Moscovici (2009). Os ciganos entre perseguição e emancipação. Sociedade e Estado, 24(3), 653-678.

Nóbrega, S., Fonte, E. \& Paula F. (2005). Do amor e da dor. Representações sociais sobre o amor e o sofrimento psíquico. Estudos de Psicologia, 22(1),7787.

Pacheco, R. O. \& Santos, S. S. C. (2004). Avaliação global de idoso em unidades de PSF. Textos sobre Envelhecimento, 7(2), 45-61.

Rosa, T. E. C., Benício, M. H. D. A. Latorre, M. R. D. O. \& Ramos, L. R. (2003). Fatores determinantes da capacidade funcional entre idosos. Revista de Saúde Pública, 37(1), 40-48.

Uchôa, E. (2003). Contribuições da antropologia para uma abordagem das questões relativas à saúde do idoso. Caderno de Saúde Pública, 19(3), 849-53.

Recebido em abril de 2010

Reformulado em setembro de 2010 Aprovado em novembro de 2010 
Sobre as autoras:

Olívia Galvão Lucena Ferreira é fisioterapeuta, especialista em Recursos Cinesioterápicos pela UFPB, mestre em Enfermagem pela UFPB e docente do curso de Fisioterapia da Faculdade de Ciências Médicas da Paraíba.

Silvana Carneiro Maciel é psicóloga, doutora em Psicologia Social, professora do Departamento de Psicologia Universidade Federal da Paraíba e do Mestrado em Psicologia Social UFPB vinculada ao Núcleo de Pesquisa Aspectos Psicossociais de Prevenção e da Saúde Coletiva e membro da ANPEPP do grupo Representações Sociais.

Antonia Oliveira Silva é enfermeira, doutora em Enfermagem Fundamental pela Escola de Enfermagem de Ribeirão Preto da Universidade de São Paulo, professora Associada da Universidade Federal da Paraíba do Departamento e da Pós-Graduação em Enfermagem e membro da ANPEPP do grupo Representações Sociais.

Roseane Christina da Nova Sá é psicóloga, doutoranda em Psicologia Social pela Universidade Federal da Paraíba, mestre em Psicologia, membro do Núcleo de Pesquisa "Aspectos Psicossociais e de Prevenção e da Saúde Coletiva”, psicóloga da Saúde (CRP 13/3729) e professora da Universidade Federal de Campina Grande - Unidade Acadêmica de Ciências da Saúde, Curso de Psicologia.

Maria Adelaide Silva P. Moreira é fisioterapeuta, doutora em Ciências da Saúde pela Universidade Federal do Rio Grande do Norte e professora Adjunta da Universidade Estadual do Sudoeste da Bahia do curso de Fisioterapia e da Pós-Graduação em Enfermagem e Saúde. 